\title{
Cosmic archaeology with gravitational waves from cosmic strings
}

\author{
Yanou Cui, ${ }^{1, *}$ Marek Lewicki, ${ }^{2,3,4, \uparrow}$ David E. Morrissey, ${ }^{5, ٪}$ and James D. Wells ${ }^{6,7, \S}$ \\ ${ }^{1}$ Department of Physics and Astronomy, University of California, Riverside, California 92521, USA \\ ${ }^{2} A R C$ Centre of Excellence for Particle Physics at the Terascale (CoEPP) \& CSSM, \\ Department of Physics, University of Adelaide, South Australia 5005, Australia \\ ${ }^{3}$ Faculty of Physics, University of Warsaw ul. Pasteura 5, $02-093$ Warsaw, Poland \\ ${ }^{4}$ Kings College London, Strand, London WC2R 2LS, United Kingdom \\ ${ }^{5}$ TRIUMF, 4004 Wesbrook Mall, Vancouver, British Columbia V6T 2A3, Canada \\ ${ }^{6}$ Leinweber Center for Theoretical Physics, University of Michigan, Ann Arbor, Michigan 48109, USA \\ ${ }^{7}$ Deutsches Elektronen-Synchrotron DESY, Theory Group, D-22603 Hamburg, Germany
}

\section{(Received 6 December 2017; published 6 June 2018)}

\begin{abstract}
Cosmic strings are generic cosmological predictions of many extensions of the standard model of particle physics, such as a $U(1)^{\prime}$ symmetry-breaking phase transition in the early Universe or remnants of superstring theory. Unlike other topological defects, cosmic strings can reach a scaling regime that maintains a small fixed fraction of the total energy density of the Universe from a very early epoch until today. If present, they will oscillate and generate gravitational waves with a frequency spectrum that imprints the dominant sources of total cosmic energy density throughout the history of the Universe. We demonstrate that current and future gravitational wave detectors, such as LIGO and LISA, could be capable of measuring the frequency spectrum of gravitational waves from cosmic strings and discerning the energy composition of the Universe at times well before primordial nucleosynthesis and the cosmic microwave background where standard cosmology has yet to be tested. This work establishes a benchmark case that gravitational waves may provide an unprecedented, powerful tool for probing the evolutionary history of the very early Universe.
\end{abstract}

DOI: 10.1103/PhysRevD.97.123505

\section{INTRODUCTION}

Gravitational waves (GW), vibrations of spacetime itself proposed by Einstein in 1916, were recently observed directly for the first time by the LIGO Collaboration [1]. The source of these signals were black hole binaries and, more recently, a neutron star binary [2]. Future measurements of such astrophysical events by the LIGO [3] and Virgo [4] detectors and the proposed LISA [5], BBO, and DECIGO [6] detectors will usher in a new era of observational astronomy and a much better understanding of the largest compact objects in the Universe.

Gravitational waves may also provide a unique test of fundamental microphysics and early Universe cosmology [7-10]. For example, primordial inflation [11], cosmic

\footnotetext{
Hanou.cui@ucr.edu

marek.lewicki@fuw.edu.pl

*dmorri@triumf.ca

§wells@umich.edu
}

Published by the American Physical Society under the terms of the Creative Commons Attribution 4.0 International license. Further distribution of this work must maintain attribution to the author(s) and the published article's title, journal citation, and DOI. Funded by SCOAP. strings [12,13], and cosmological first-order phase transitions [14-16] are all expected to create GWs. While the GWs from inflation are typically below the sensitivity of current and planned future detectors [17-19] (although see Ref. [20]), GWs from cosmic strings or phase transitions can produce observable signals. In many cases, the potential sensitivity of GW detectors to these phenomena extends well beyond the reach of other foreseeable laboratory and cosmological tests. In this paper, we focus on the GWs from cosmic strings and demonstrate their unique potential for exploring cosmological history.

Cosmic strings are stable one-dimensional objects characterized by a tension $\mu$. They arise in superstring theory as fundamental or $(p, q)$ strings [21,22]. They can also emerge as vortexlike solutions of field theory [23], such as configurations that wrap one or more times at spatial infinity in theories with a spontaneously broken $U(1)$, in which case the tension is related to the symmetry-breaking scale by $\mu \sim \sigma^{2}$ [24]. In the cosmological setting, cosmic strings form a network of horizon-length long strings together with a collection of closed string loops [12,13]. Such a network would distort the cosmic microwave background (CMB), and current observations limit $G \mu<1.1 \times 10^{-7}$, where $G$ is Newton's constant [25]. 
Gravitational radiation is a key part of the evolution of a cosmic string network $[12,13]$. Long strings intercommute and reconnect to create closed string loops. These loops then oscillate, emitting energy in the form of gravitational waves (and sometimes other forms of radiation) until they decay away [26-29]. Together, the processes of intercommutation, oscillation, and emission allow the string network to shed energy efficiently and prevent it from dominating the energy density of the Universe. Instead, a cosmic string network is expected to reach a scaling regime in which it tracks the total energy density with fraction on the order of $G \mu$ [30-32]. The scaling property of cosmic strings leads to a stochastic background of gravitational radiation built up from GW emission over the history of the string network $[26,33]$.

In this paper, we show that the frequency spectrum of GWs from cosmic strings can be used to look back in time and test the evolutionary history of the Universe. For each frequency band of the background observed today, the emission was dominated by strings in a particular era of the early Universe [33]. The standard thermal picture for the evolution of the cosmos is primordial inflation followed by reheating to a high temperature, and a subsequent long period in which the expansion of the Universe is driven by a dominant energy density of radiation until the more recent transitions to matter and then dark energy domination. Evidence for this "standard cosmology" comes primarily from observations of the CMB [34] and the successful predictions of big bang nucleosynthesis (BBN), corresponding to cosmic temperatures up to $T \simeq 5 \mathrm{MeV}$ [35]. Measurements of the $\mathrm{GW}$ frequency spectrum from cosmic strings by current and planned detectors could test the standard cosmology at even earlier times and possibly reveal deviations from it.

To demonstrate the power of GWs from cosmic strings to probe the very early Universe, we study the frequency spectrum emitted by a string network in the standard cosmology and in two well-motivated variations. We focus on an ideal Nambu-Goto (NG) cosmic string network with unit reconnection probability and loops that radiate exclusively into GWs, and we apply the results of recent simulations of string networks to compute the GW spectrum. We show that a combination of current and planned GW detectors with different frequency sensitivities may enable us to reconstruct a timeline of cosmic history well beyond the BBN epoch.

\section{GW FROM COSMIC STRINGS}

Oscillating closed string loops are typically the dominant source of GWs from a cosmic string network in the scaling regime. The length $\ell$ of a string loop created by the network at time $t_{i}$ evolves according to

$$
\ell=\alpha t_{i}-\Gamma G \mu\left(t-t_{i}\right) .
$$

The first term is the initial loop size as a fraction $\alpha$ of the formation time $t_{i}$, i.e., a fraction of the horizon size. Recent cosmic string simulations find that about $10 \%$ of the energy released by the long string network goes to $\alpha \simeq 10^{-1}$ large loops, with the remaining $90 \%$ going to the kinetic energies of highly boosted smaller loops [36-42]. The kinetic energy redshifts away and is not transferred to GWs. The second term above describes the shortening of the loop as it emits gravitational radiation, assumed to be the only radiation emitted, and characterized by the dimensionless constant $\Gamma \simeq 50[26,28,41-43]$.

String loops emit GWs from normal mode oscillations at frequencies $f_{\text {emit }}=2 k / \ell, k \in \mathbb{Z}^{+}$. After emission, the frequency of the $\mathrm{GW}$ redshifts as $a^{-1}$, where $a(t)$ is the cosmological scale factor. For a given GW frequency $f$ observed today from mode $k$, this implies the emission time $\tilde{t}$ is related to the loop formation time by

$$
f=\frac{a(\tilde{t})}{a\left(t_{0}\right)} \frac{2 k}{\alpha t_{i}-\Gamma G \mu\left(\tilde{t}-t_{i}\right)},
$$

where $t_{0}$ is the current time.

The stochastic GW background depends on the rate of loop production by the cosmic string network. We model this using the velocity-dependent one-scale (VOS) model [44-46], with a loop chopping efficiency of $\bar{c}=0.23$ [46]. Applying local energy conservation and considering only large loops, this yields a loop formation rate per unit volume at time $t_{i}$ of

$$
\frac{d n_{\text {loop }}}{d t_{i}}=(0.1) \frac{C_{\text {eff }}\left(t_{i}\right)}{\alpha} t_{i}^{-4},
$$

where the first factor accounts for the $10 \%$ of the network energy going to large loops discussed earlier $[41,42]$. The function $C_{\text {eff }}\left(t_{i}\right)$ depends on the redshift scaling of the dominant energy density $\rho$ of the Universe. When $\rho$ is dominated by a single source, it scales approximately as

$$
\rho \propto a^{-n}
$$

For $n=3$ (matter domination), 4 (radiation domination), and 6 (kination-to be explained later), the VOS model predicts $C_{\text {eff }}=0.41,5.5,30$, respectively.

Summing over all harmonic modes, the GW density per unit frequency seen today is

$$
\Omega_{\mathrm{GW}}(f)=\frac{f}{\rho_{c}} \frac{d \rho_{\mathrm{GW}}}{d f}=\sum_{k} \Omega_{\mathrm{GW}}^{(k)}(f),
$$

with

$$
\begin{aligned}
\Omega_{\mathrm{GW}}^{(k)}(f)= & \frac{1}{\rho_{c}} \frac{2 k}{f} \frac{(0.1) \Gamma_{k} G \mu^{2}}{\alpha(\alpha+\Gamma G \mu)} \\
& \times \int_{t_{F}}^{t_{0}} d \tilde{t} \frac{C_{\mathrm{eff}}\left(t_{i}\right)}{t_{i}^{4}}\left[\frac{a(\tilde{t})}{a\left(t_{0}\right)}\right]^{5}\left[\frac{a\left(t_{i}\right)}{a(\tilde{t})}\right]^{3} \Theta\left(t_{i}-t_{F}\right),
\end{aligned}
$$


where $\rho_{c}=3 H_{0}^{2} / 8 \pi G$ is the critical density, $\Gamma_{k}=$ $\Gamma /\left(3.60 \cdot k^{4 / 3}\right) \quad[41,42], t_{i}$ is obtained by inverting Eq. (2), and $t_{F}$ is the formation time of the string network.

In Fig. 1, we show the frequency spectrum of GWs $\Omega_{\mathrm{GW}} h^{2}$ from cosmic strings for $G \mu=5 \times 10^{-12}, \alpha=10^{-1}$ and a standard cosmological history. Also shown are the current sensitivity bands of LIGO $[1,3,47]$ and the projected sensitivities of LISA [48] and DECIGO/BBO [6]. The upper left solid triangle indicates the current limit from timing measurements by the European Pulsar Timing Array (EPTA) [49], with the expected sensitivity of the future Square Kilometre Array (SKA) [50] shown below. The EPTA limit implies $G \mu \lesssim 10^{-11}$ giving the strongest current bound on the cosmic string network. Our results are consistent with the recent calculations of Refs. [51,52].

Figure 1 illustrates the key relationship between the GW frequency spectrum and the cosmological era at which a given frequency today was produced. At higher frequencies, where the relic GW spectrum would normally be dominated by loops produced in the radiation era, the result of Eq. (6) is approximately independent of $\alpha$ and scales as

$$
\Omega_{\mathrm{GW}}(f) \propto\left\{\begin{array}{ll}
f^{\frac{8-2 n}{2-n}} & n>10 / 3 \\
f^{-1} & n \leq 10 / 3
\end{array} .\right.
$$

This yields a flat spectrum for radiation and a $1 / f$ spectrum for matter. At lower frequencies, the scaling (for $\alpha \gg \Gamma G \mu$ ) goes like $f^{3 / 2}$. The characteristic flatness of the spectrum at higher frequencies from early radiation domination implies that deviations from this scenario would be dramatic.

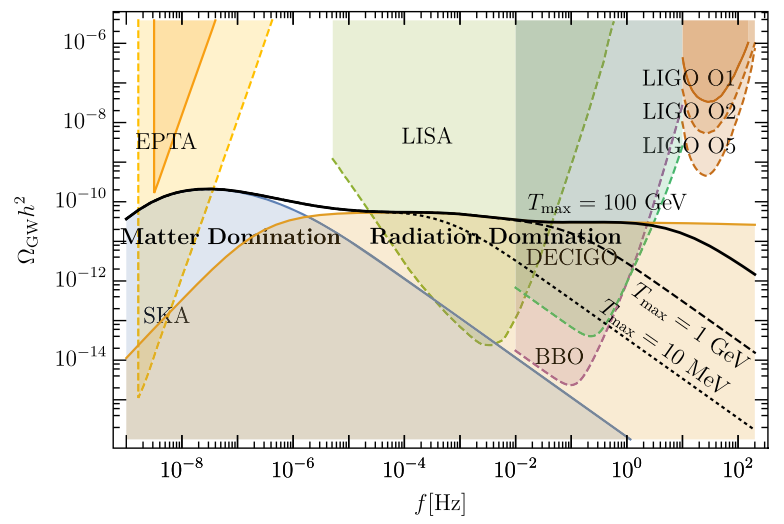

FIG. 1. Frequency spectrum $\Omega_{\mathrm{GW}} h^{2}$ of GWs from a cosmic string network with $G \mu=5 \times 10^{-12}$ and $\alpha=10^{-1}$ assuming a standard cosmological history. The blue (orange) region indicates contributions emitted during matter (radiation) domination with $n=3$ ( $n \simeq 4)$. The falling black lines indicate the effect on the spectrum if only those loops created below the temperatures $T_{\max }=10 \mathrm{MeV}$ (dotted), $T_{\max }=1 \mathrm{GeV}$ (dashed), and $T_{\max }=$ $100 \mathrm{GeV}$ (solid) contribute. The upper shaded regions show current and future experimental GW sensitivities.

\section{TESTS OF THE STANDARD COSMOLOGY}

Cosmic string scaling implies that the relic spectrum of GWs today originated from an extended period of evolution of the early Universe. As a result, the GW spectrum from cosmic strings can test the standard picture of a Universe dominated by radiation following inflation and reheating, and probe deviations from it. To illustrate this, we compare the predictions of the standard picture to two wellmotivated nonstandard histories. The first is a transient period of matter domination prior to the standard radiation era, corresponding to $n=3$ in Eq. (4). This can arise from a large density of a long-lived massive particle or from the oscillation of a scalar moduli field in a quadratic potential [53]. Matter dominance typically ends during a reheating phase in which the relevant species decays to light Standard Model (SM) particles. Our second example of nonstandard cosmology is a period of "kination", with $n>4$ in Eq. (4). This can arise from the oscillation of a scalar field in a nonquadratic potential: for $V(\phi) \propto \phi^{N}$ one obtains $n=6 N /(N+2)$, which can occur in quintessence models for dark energy or inflation [54,55]. We focus on the specific case of $n=6$ since it provides the upper envelope for the effect of all $n>4$.

For both nonstandard scenarios, let $t_{\Delta}$ be the time at which the Universe transitions to the standard period of radiation domination. The evolution of the energy density of the Universe during and after the nonstandard phase can be parametrized according to

$$
\rho(t)= \begin{cases}\rho_{\mathrm{st}}\left(t_{\Delta}\right)\left[\frac{a\left(t_{\Delta}\right)}{a(t)}\right]^{n} & ; t<t_{\Delta} \\ \rho_{\mathrm{st}}(t) & ; t \geq t_{\Delta}\end{cases}
$$

where $\rho_{\mathrm{st}}$ is the energy density extrapolated assuming the standard cosmological history, and $n=6(n=3)$ for early kination (matter) domination. We also define $T_{\Delta}$ as the temperature at time $t_{\Delta}$ when radiation domination resumes. In scenarios with early matter domination, $T_{\Delta}$ coincides with the reheating temperature. For both the matterdominated and kination scenarios, $T_{\Delta} \gtrsim 5 \mathrm{MeV}$ is needed for consistency with BBN [56].

In Fig. 2, we compare the frequency spectrum of GWs from a cosmic string network with $G \mu=5 \times 10^{-15}$, $5 \times 10^{-17}$ and a standard cosmological history to the corresponding spectra for two nonstandard histories with $n=3,6$ and $T_{\Delta}=5 \mathrm{MeV}$. Also shown are the experimental sensitivities of current and future detectors as in Fig. 1. The deviations in the GW spectra shown in Fig. 2 due to nonstandard cosmologies are dramatic and potentially distinguishable from the nearly flat spectrum from the standard cosmology at future GW detectors. As expected from Eq. (7), the GW spectrum grows with $f$ for $n>4$ and falls with $f$ for $n<4$. Relative to the flat spectrum from $n=4$, this makes an early kination $(n=6)$ phase easier to observe, and we find current LIGO data already implies 


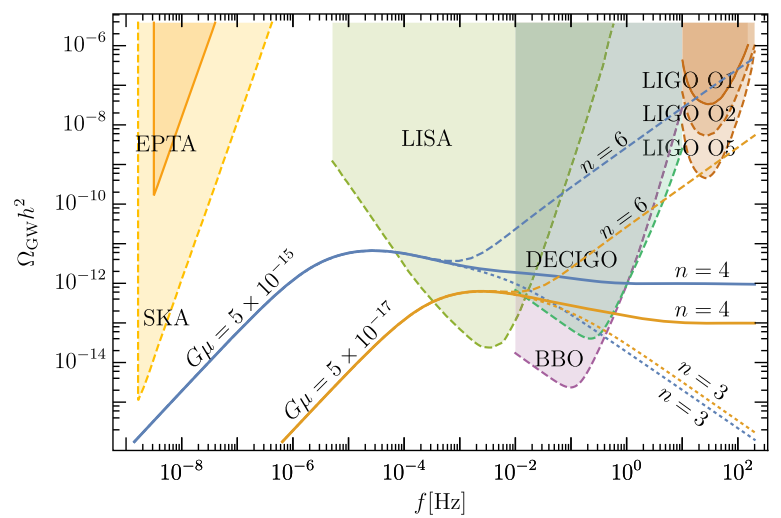

FIG. 2. Gravitational wave frequency spectra $\Omega_{\mathrm{GW}} h^{2}(f)$ from a cosmic string network with $G \mu=5 \times 10^{-15}$ (blue), $5 \times 10^{-17}$ (orange) and $\alpha=10^{-1}$ for standard and nonstandard cosmological histories. The solid lines show the spectra for the standard evolution while the dashed (dotted) lines show the GW spectra for an early $n=6$ kination ( $n=3$ matter) era ending at temperature $T_{\Delta}=5 \mathrm{MeV}$. The upper shaded regions indicate the current and future sensitivities of GW detectors and pulsar timing arrays.

a limit of $G \mu \lesssim 5 \times 10^{-15}$ for $T_{\Delta}=5 \mathrm{MeV}$, orders of magnitude smaller than other bounds on the string network. In contrast, an early matter phase $(n=3)$ leads to a characteristic downturn in the GW spectrum at high frequency that is still potentially observable by future detectors such as LISA, DECIGO, and BBO.

An additional constraint not yet included comes from the total radiation density of GWs, which must not exceed the limits from the CMB and BBN. This translates into the bound $[57,58]$

$$
\int d(\ln f) \Omega_{\mathrm{GW}} \lesssim 3.8 \times 10^{-6}
$$

For standard early radiation domination, this places a moderate constraint on $\Omega_{\mathrm{GW}}$ with a logarithmic sensitivity to the highest frequencies created in this era. The bound becomes more severe for $n>4$ since the relic GW spectrum now increases with frequency as a power law, Eq. (7). This growth is expected to be cut off at a high frequency that corresponds to the onset of the $n>4$ phase or the creation of the string network. In the early $n=6$ scenario with $T_{\Delta}=5 \mathrm{MeV}$, the maximal temperature in this phase is $T \simeq$ $4 \mathrm{GeV}(20 \mathrm{GeV})$ for $G \mu=5 \times 10^{15}\left(5 \times 10^{-17}\right)$.

As can be seen in Fig. 2, the deviation in the GW spectrum due to a nonstandard cosmic history corresponds to a departure from approximate flatness at a characteristic frequency $f_{\Delta}$ that depends on the string network parameters and $T_{\Delta}$, but is nearly independent of the energy redshift exponent $n$. In Fig. 3, we show $f_{\Delta}$ as a function of $T_{\Delta}$ for several values of $G \mu$. These curves follow the approximate relation $f_{\Delta} \propto T_{\Delta}(G \mu)^{-\frac{1}{2}} \alpha^{-\frac{1}{2}}$ (valid for $\alpha \gg \Gamma G \mu$ ), which we

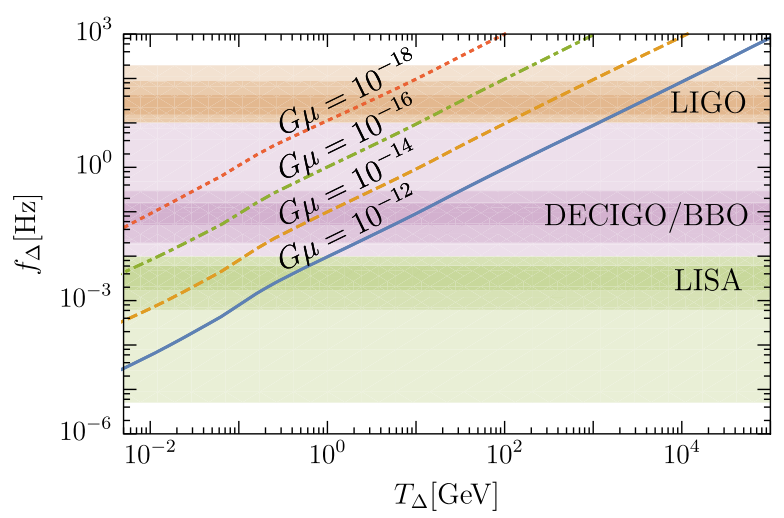

FIG. 3. Frequency $f_{\Delta}$ at which the GW spectrum from cosmic strings is altered by a nonstandard cosmology as a function of $T_{\Delta}$ for several values $G \mu$ and $\alpha=10^{-1}$. The shaded bands indicate the sensitivities of current and future GW detectors, with the darker regions showing the peak sensitivities.

derive in a future work [59]. Roughly speaking, $T_{\Delta}$ characterizes the formation time of loops that produce the dominant contribution to GWs with frequency $f_{\Delta}$ today. Figure 3 also shows the frequency sensitivity bands of various $\mathrm{GW}$ detectors relative to $f_{\Delta}$ (but note that the amplitude may be below the corresponding detector sensitivity).

\section{DISCUSSION}

If cosmic strings are realized in nature, they could provide a unique and powerful tool for probing the history of the early Universe. We have demonstrated that the frequency spectrum of GWs emitted by a cosmic string network depends dramatically on the energy content of the Universe when they are produced. Current and planned experiments will have the potential to measure such a spectrum and thereby test the evolution of the cosmos at much earlier times than ever before.

The reach of this method for looking back in time depends on the frequency sensitivity of GW detectors and the properties of the cosmic string network. In general, deviations from the standard cosmological evolution at earlier times imprint themselves on the GW spectrum at higher frequencies, as shown in Fig. 3. These considerations provide strong motivation to explore new methods to extend the sensitivity of future GW observatories to higher frequencies beyond LIGO [60-62] and further motivate searches for memory effects of high frequency GW signals [63].

The frequency spectrum of GWs also depends on the distribution of string loop sizes. Based on cosmic string simulations [41,42], we have modeled this by assuming that $10 \%$ of the energy shed by a scaling string network goes to loops with a fixed initial loop size parameter of $\alpha=10^{-1}$. Our simple prescription reproduces the radiation-era loop length distribution found in the simulation of Ref. [40,41] 
and is similar to their result for the matter era, but it is also optimistic. Smaller values of $\alpha$ would push the spectral features of nonstandard cosmologies to higher frequencies and potentially outside the range of GW detectors. Let us also mention that we do not know of any simulations of loop formation or scaling during a kination phase, which we have assumed in our calculation. Our results could also be modified in theories with more complex cosmic string dynamics such as $(p, q)$ strings with small intercommutation probabilities [64] or in scenarios where strings emit nongravitational radiation [65-70].

Other potential sources of gravitational waves imply two important further questions. First, we have not considered the expected GW background from unresolved astrophysical events, such as compact binary mergers [71]. These could be large enough to be observed at LIGO and will present a challenging background to stochastic cosmological signals of lower amplitude [72,73]. However, studies of LISA [74], DECIGO/BBO [6,75], and future ground-based GW arrays [76] suggest that improvements in spatial resolution and analysis methods [77] will allow such backgrounds to be removed to a very high degree. A second question is whether other cosmological sources of GWs could mimic those from cosmic strings with the standard cosmology. In general, the flat spectrum from cosmic strings appears to be very distinctive and difficult to reproduce with a single source. Much more difficult would be to distinguish the spectrum from cosmic strings with a modified cosmology from other sources, but even here a spectrum that is flat in frequency for at least a decade or two would be strongly suggestive of a string origin.
In this paper, we have investigated the effects of nonstandard cosmological histories on the frequency spectrum of stochastic GWs produced by cosmic strings. We have demonstrated that cosmic string GWs could provide an unprecedented window on the evolution of the very early Universe prior to $\mathrm{BBN}$ and the $\mathrm{CMB}$. This work may serve as an inspiring benchmark for exploiting the full potential of GW as a new tool for probing particle physics and cosmology beyond the horizon of our current knowledge.

\section{ACKNOWLEDGMENTS}

We would like to thank Masha Baryakhtar and Mairi Sakellariadou for interesting discussions. The work of M. L. was supported by the Australian research Council (ARC) Centre of Excellence for Particle Physics at the Terascale (CoEPP) (CE110001104), the Centre for the Subatomic Structure of Matter (CSSM), the Polish Ministry of Science and Higher Education (MNiSW) Grant No. IP2015 043174 and Science and Technology Facilities Council (STFC) Grant No. ST/L000326/1. D. M. is supported by a Discovery Grant from the Natural Sciences and Engineering Research Council of Canada (NSERC), and TRIUMF, which receives federal funding via a contribution agreement with the National Research Council of Canada (NRC). J. D. W. was supported in part by the Department of Energy Grant No. DE-SC0007859 and the Humboldt Research Award of the Humboldt Foundation.
[1] B. P. Abbott et al., Phys. Rev. Lett. 116, 061102 (2016).

[2] B. P. Abbott et al. (Virgo, LIGO Scientific), Phys. Rev. Lett. 119, 161101 (2017).

[3] J. Aasi et al. (LIGO Scientific), Classical Quantum Gravity 32, 115012 (2015).

[4] F. Acernese et al. (VIRGO), Classical Quantum Gravity 32, 024001 (2015).

[5] H. Audley et al., arXiv:1702.00786.

[6] K. Yagi and N. Seto, Phys. Rev. D 83, 044011 (2011).

[7] P. Binetruy, A. Bohe, C. Caprini, and J.-F. Dufaux, J. Cosmol. Astropart. Phys. 06 (2012) 027.

[8] A. Arvanitaki, M. Baryakhtar, S. Dimopoulos, S. Dubovsky, and R. Lasenby, Phys. Rev. D 95, 043001 (2017).

[9] S. Bird, I. Cholis, J. B. Muoz, Y. Ali-Hamoud, M. Kamionkowski, E. D. Kovetz, A. Raccanelli, and A. G. Riess, Phys. Rev. Lett. 116, 201301 (2016).

[10] D. Croon, A. E. Nelson, C. Sun, D. G. E. Walker, and Z.-Z. Xianyu, arXiv:1711.02096.

[11] L. F. Abbott and M. B. Wise, Nucl. Phys. B244, 541 (1984).
[12] M. B. Hindmarsh and T. W. B. Kibble, Rep. Prog. Phys. 58, 477 (1995).

[13] A. Vilenkin and E. P.S. Shellard, Cosmic Strings and Other Topological Defects (Cambridge University Press, Cambridge, England, 2000).

[14] A. Kosowsky, M. S. Turner, and R. Watkins, Phys. Rev. Lett. 69, 2026 (1992).

[15] M. Kamionkowski, A. Kosowsky, and M. S. Turner, Phys. Rev. D 49, 2837 (1994).

[16] D. J. Weir, arXiv:1705.01783.

[17] T. L. Smith, M. Kamionkowski, and A. Cooray, Phys. Rev. D 73, 023504 (2006).

[18] K. N. Ananda, C. Clarkson, and D. Wands, Phys. Rev. D 75, 123518 (2007).

[19] M. C. Guzzetti, N. Bartolo, M. Liguori, and S. Matarrese, Riv. Nuovo Cimento 39, 399 (2016).

[20] P. D. Lasky et al., Phys. Rev. X 6, 011035 (2016).

[21] E. J. Copeland, R. C. Myers, and J. Polchinski, J. High Energy Phys. 06 (2004) 013. 
[22] G. Dvali and A. Vilenkin, J. Cosmol. Astropart. Phys. 03 (2004) 010.

[23] H. B. Nielsen and P. Olesen, Nucl. Phys. B61, 45 (1973).

[24] T. W. B. Kibble, J. Phys. A 9, 1387 (1976).

[25] T. Charnock, A. Avgoustidis, E. J. Copeland, and A. Moss, Phys. Rev. D 93, 123503 (2016).

[26] A. Vilenkin, Phys. Lett. 107B, 47 (1981).

[27] T. Vachaspati and A. Vilenkin, Phys. Rev. D 31, 3052 (1985).

[28] N. Turok, Nucl. Phys. B242, 520 (1984).

[29] C. J. Burden, Phys. Lett. 164B, 277 (1985).

[30] A. Albrecht and N. Turok, Phys. Rev. Lett. 54, 1868 (1985).

[31] D. P. Bennett and F. R. Bouchet, Phys. Rev. Lett. 60, 257 (1988).

[32] B. Allen and E. P. S. Shellard, Phys. Rev. Lett. 64, 119 (1990).

[33] R. R. Caldwell and B. Allen, Phys. Rev. D 45, 3447 (1992).

[34] P. A. R. Ade et al. (Planck), Astron. Astrophys. 594, A13 (2016).

[35] R. H. Cyburt, B. D. Fields, K. A. Olive, and T.-H. Yeh, Rev. Mod. Phys. 88, 015004 (2016).

[36] V. Vanchurin, K. D. Olum, and A. Vilenkin, Phys. Rev. D 74, 063527 (2006).

[37] K. D. Olum and V. Vanchurin, Phys. Rev. D 75, 063521 (2007).

[38] C. J. A. P. Martins and E. P. S. Shellard, Phys. Rev. D 73, 043515 (2006).

[39] C. Ringeval, M. Sakellariadou, and F. Bouchet, J. Cosmol. Astropart. Phys. 02 (2007) 023.

[40] J. J. Blanco-Pillado, K. D. Olum, and B. Shlaer, Phys. Rev. D 83, 083514 (2011).

[41] J. J. Blanco-Pillado, K. D. Olum, and B. Shlaer, Phys. Rev. D 89, 023512 (2014).

[42] J. J. Blanco-Pillado and K. D. Olum, Phys. Rev. D 96, 104046 (2017).

[43] J. M. Quashnock and D. N. Spergel, Phys. Rev. D 42, 2505 (1990).

[44] C. J. A. P. Martins and E. P. S. Shellard, Phys. Rev. D 53, R575 (1996).

[45] C. J. A. P. Martins and E. P. S. Shellard, Phys. Rev. D 54, 2535 (1996).

[46] C. J. A. P. Martins and E. P. S. Shellard, Phys. Rev. D 65, 043514 (2002).

[47] E. Thrane and J. D. Romano, Phys. Rev. D 88, 124032 (2013).

[48] N. Bartolo et al., J. Cosmol. Astropart. Phys. 12 (2016) 026.

[49] R. van Haasteren et al., Mon. Not. R. Astron. Soc. 414, 3117 (2011); 425, 1597(E) (2012).

[50] G. Janssen et al., Proc. Sci. AASKA14 (2015) 037 [arXiv: 1501.00127].
[51] J. J. Blanco-Pillado, K. D. Olum, and X. Siemens, Phys. Lett. B 778, 392 (2018).

[52] C. Ringeval and T. Suyama, arXiv:1709.03845.

[53] T. Moroi and L. Randall, Nucl. Phys. B570, 455 (2000).

[54] P. Salati, Phys. Lett. B 571, 121 (2003).

[55] D. J. H. Chung, L. L. Everett, and K. T. Matchev, Phys. Rev. D 76, 103530 (2007).

[56] S. Hannestad, Phys. Rev. D 70, 043506 (2004).

[57] S. Henrot-Versille et al., Classical Quantum Gravity 32, 045003 (2015).

[58] T. L. Smith, E. Pierpaoli, and M. Kamionkowski, Phys. Rev. Lett. 97, 021301 (2006).

[59] Y. Cui, M. Lewicki, D. E. Morrissey, and J. D. Wells (to be published).

[60] A. Arvanitaki and A. A. Geraci, Phys. Rev. Lett. 110, 071105 (2013).

[61] A. S. Chou et al. (Holometer), Phys. Rev. Lett. 117, 111102 (2016).

[62] B. P. Abbott et al. (LIGO Scientific), Classical Quantum Gravity 34, 044001 (2017).

[63] L. O. McNeill, E. Thrane, and P. D. Lasky, Phys. Rev. Lett. 118, 181103 (2017).

[64] M. G. Jackson, N. T. Jones, and J. Polchinski, J. High Energy Phys. 10 (2005) 013.

[65] M. Srednicki and S. Theisen, Phys. Lett. B 189, 397 (1987).

[66] A. Vilenkin and T. Vachaspati, Phys. Rev. Lett. 58, 1041 (1987).

[67] T. Damour and A. Vilenkin, Phys. Rev. Lett. 78, 2288 (1997).

[68] Y. Cui, S. P. Martin, D. E. Morrissey, and J. D. Wells, Phys. Rev. D 77, 043528 (2008).

[69] Y. Cui and D. E. Morrissey, Phys. Rev. D 79, 083532 (2009).

[70] A. J. Long, J. M. Hyde, and T. Vachaspati, J. Cosmol. Astropart. Phys. 09 (2014) 030.

[71] T. Regimbau, Res. Astron. Astrophys. 11, 369 (2011).

[72] B. P. Abbott et al. (Virgo, LIGO Scientific), Phys. Rev. Lett. 118, 121101 (2017); Phys. Rev. Lett. 119, 029901(E) (2017).

[73] B. P. Abbott et al. (Virgo, LIGO Scientific), Phys. Rev. Lett. 120, 091101 (2018).

[74] R. Umstatter, N. Christensen, M. Hendry, R. Meyer, V. Simha, J. Veitch, S. Vigeland, and G. Woan, Phys. Rev. D 72, 022001 (2005).

[75] J. Harms, C. Mahrdt, M. Otto, and M. Priess, Phys. Rev. D 77, 123010 (2008).

[76] T. Regimbau, M. Evans, N. Christensen, E. Katsavounidis, B. Sathyaprakash, and S. Vitale, Phys. Rev. Lett. 118, 151105 (2017).

[77] A. Jenkins and M. Sakellariadou, arXiv:1802.06046. 